Review Article

\title{
Human Mesenchymal Stromal Cell-Mediated Immunoregulation: Mechanisms of Action and Clinical Applications
}

\author{
Akaitz Dorronsoro, Jon Fernández-Rueda, Karoline Fechter, Izaskun Ferrin, \\ Juan Manuel Salcedo, Emma Jakobsson, and César Trigueros
}

Mesenchymal and Hematopoietic Stem Cell Department, Fundación Inbiomed, Foundation for Stem Cell Research, Paseo Mikeletegi 81, 20009 San Sebastián, Spain

Correspondence should be addressed to César Trigueros; ctrigueros@inbiomed.org

Received 25 June 2013; Accepted 30 August 2013

Academic Editor: David A. Rizzieri

Copyright (C) 2013 Akaitz Dorronsoro et al. This is an open access article distributed under the Creative Commons Attribution License, which permits unrestricted use, distribution, and reproduction in any medium, provided the original work is properly cited.

Mesenchymal stromal cells (MSCs) are multipotent cells found in connective tissues that can differentiate into bone, cartilage, and adipose tissue. Interestingly, they can regulate immune responses in a paracrine way and allogeneic MSCs do not elicit immune response. These properties have encouraged a number of clinical trials in a broad range of regenerative therapies. Although these trials were first focused on their differentiation properties, in the last years, the immunosuppressive features have gained most of the attention. In this review, we will summarize the up-to-date knowledge about the immunosuppressive mechanisms of MSCs in vivo and in vitro and the most promising approaches in clinical investigation.

\section{Introduction}

Mesenchymal stromal cells (MSCs) are found in a variety of tissues, although bone marrow represents the most common source for research and clinical purposes. These cells are multipotent progenitors that have the capacity to differentiate into multiple lineages such as bone, cartilage, and adipocytes [1-3]. MSCs have received renewed interest in the last five years, particularly due to their ability to modulate the immune response. This property, in combination with the facts that they are not immunogenic and preferentially home to damaged tissue, makes them good candidates for a therapeutic approach of cell-based therapy for a wide range of autoimmune disorders [4-6]. Currently, there are a large number of ongoing clinical trials employing MSCs as immunomodulators.

MSCs have been shown to regulate the activity in a range of effector cells involved in both innate and adaptive immunities. The crosstalk between MSCs and the cells of the immune system leads to an increased production of several soluble immunomodulatory factors. Despite identification of many of these factors, the mechanism behind MSCs immunomodulation is not fully understood. However, the inflammatory environment and in particular the immune cells involved in each phase of the immune response are likely to be the critical determinants of the regulatory process. The immunosuppressive ability of MSCs is not constitutive but rather is induced by crosstalk with cells of the immune system [7-10]. Therefore, different inflammation status might lead to distinct immunomodulatory responses. This is a fundamental concept that could determine future clinical settings: treatment dose, timing, and frequency of administration, as well as the choice of the source of MSCs.

\section{MSC-Mediated \\ In Vitro Immunosuppression}

The ability of MSCs to modulate several processes of the immune system in vitro has been intensively studied in the last years. MSCs have a broad range of target immune effector cells and are able to inhibit key functions of innate 
and adaptive immune cells during inflammatory responses. The exact mechanisms by which MSCs are able to regulate immune functions are still not fully understood. However, while the requirement of cell-to-cell contact is not clear, a number of soluble factors involved in the process have been identified.

The most studied soluble molecules and cytokines secreted by MSCs and involved in immunosuppression are indoleamine 2,3-dioxygenase (IDO), prostaglandin E2 (PGE2), transforming growth factor-beta (TGF- $\beta$ ), hepatocyte growth factor (HGF), and interleukin-10 (IL-10). Furthermore, in the past few years, the implication of other molecules like human leukocyte antigen-G (HLA-G5) has been shown to be associated with the immunomodulatory properties of MSC.

2.1. T Cells. MSC-mediated inhibition of $\mathrm{T}$ cell proliferation has been largely described. In vitro, MSCs can inhibit $\mathrm{T}$ cell proliferation regardless of the signaling pathway stimulated in the lymphocytes (i.e., alloantigen-, mitogen-, or anti-CD3/anti-CD28-mediated stimulation) [11, 12]. This immunomodulation does not require antigen match, as it has been shown to have similar efficiencies in cocultures of MSCs and $\mathrm{T}$ cells from the same donor or from different donors. Upon coculture, MSCs induce a G0/G1 checkpoint arrest in $\mathrm{T}$ cells, but whether this leads to a permanent arrest or if they can resume proliferation once separated from MSCs remains unclear $[12,13]$.

The mechanisms by which MSCs are able to mediate immunosuppression of $\mathrm{T}$ cells are diverse and complex, and several secreted effector molecules have been linked to this process. Among them, IDO, PGE2, TGF- $\beta$, and HGF have been described to play a major role.

IDO is the first and rate-limiting enzyme in tryptophan metabolism catalyzing the breakdown of tryptophan to $\mathrm{N}$-formylkynurenine. It is known that both tryptophan starvation and the presence of $\mathrm{N}$-formylkynurenine induce proliferative arrest of T cells $[14,15]$. In MSCs, IDO is not constitutively expressed, but upon stimulation with inflammatory cytokines, mainly by IFN- $\gamma$, the transcription is increased significantly [16]. Once IDO is expressed in the MSC, this enzyme catalyzes the breakdown of tryptophan and induces the immunomodulative effect of MSCs on T cells [17].

PGE2 is synthesized from fatty acids by cyclooxygenases 1 and 2 (COX-1, COX-2), and its immunosuppressive effects on $\mathrm{T}$ cells have been largely studied. PGE2 reduces interleukin- 2 (IL-2) and interferon-gamma (IFN- $\gamma$ ) secretion and increases cyclic adenosine monophosphate (cAMP) levels, leading to the inhibition of $\mathrm{T}$ cell proliferation [18]. PGE2 is constitutively produced by MSCs and proinflammatory factors increase its secretion. Chemical inhibition of COX-2 in MSCs and $\mathrm{T}$ cell cocultures reduces PGE2 levels, resulting in a partial impairment of MSC-driven immunosuppression [19].

The role of TGF- $\beta$ in immunosuppression is still not fully known. TGF- $\beta$ is constitutively secreted by MSCs, and its expression is notably increased in the presence of peripheral blood mononuclear cells (PBMCs). Although different TGF- $\beta$ isoforms have been linked to immunoregulation, as shown by the addition of neutralizing antibodies to cocultures or mixed lymphocyte reaction (MLR) alone [12, 20], other publications rule out this function [21].

To which extent HGF, constrictively expressed in MSC [16], plays a role in MSC-mediated immunosuppression still remains unclear. While some authors point out a role for this factor alone or demonstrating additive effect together with TGF- $\beta[12,20]$, others discard its function in immunoregulation, rather attributing its existence in cocultures to its production by lymphocytes [21].

Finally, HLA-G5, a nonclassical HLA class I molecule, has first been shown in the maternal tolerance to the fetus by mediating inhibition of NK cell cytotoxicity. Since then, its role in immunoregulation has been described in various types of pathological conditions such as viral infections, tumors, autoimmune diseases, and solid organ transplantation [38]. HLA-G5 is expressed on and secreted by adult MSCs, and its expression is linked to the immunosuppressive effects of MSCs on activated T cells via a mechanism including $\mathrm{CD} 4^{+} \mathrm{CD} 25^{+}$Foxp $^{+}{ }^{+}$regulatory T cells and IL-10 [39-41].

2.2. Natural Killer Cells. The relationship between MSCs and NK cells is ambiguous, and the mechanisms by which MSCs regulate inflammatory functions of NK cells are not well understood. On the one hand, freshly isolated NK cells fail to attack MSCs, while in contrast in vitro preactivated NK cells acquire this ability [42]. On the other hand, MSCs can inhibit proliferation, cytokine secretion and cytotoxicity of NK cells. PGE2 and TGF- $\beta$ secretion, by MSCs has been linked to antiproliferative effects and reduction in cytokine production. Data suggest that cell-to-cell contact is also involved in this type of immunomodulatory effect. Several groups have demonstrated the ability of MSCs to impair the proliferation of NK cells activated by IL-2 and interleukin-15 (IL-15). Secretion of IFN- $\gamma$, tumor necrosis factor-alpha (TNF- $\alpha$ ), and IL-10 by activated NK cells is also reduced by MSCs in vitro, in cell-to-cell contact conditions and independently of the contact mechanisms. Moreover, MSCs are able to inhibit the cytotoxic activity of NK cells when they are targeted against major histocompatibility complex-I positive (MHCI), but not MHC-I negative tumors [19, 43, 44].

2.3. B Cells. The effect of human MSCs on B cells mainly depends on several factors linked to B cell biology, the state of differentiation, and the kind and strength of stimulus given to the cells. Furthermore, results depend on dissection of viability, proliferation, and differentiation of $B$ cells and show strong dependence on methodological differences. While some studies show that MSCs inhibit B cell proliferation, others claim that this effect is not statistically significant, or even that MSCs stimulate B cell proliferation [45-48]. The same is true for immunoglobulin (Ig) secretion studies where results range from inhibition to increase [45] of secretion of IgA, IgG, or IgM by MSCs [46].

2.4. Monocytes. The role of MSCs in immunoregulation of monocytes is considered to affect both different maturation steps and their function as antigen-presenting cells 
(APCs). First, MSCs impair the differentiation and maturation towards effector dendritic cells (DCs). They can induce a reduction in the expression of CD1a, CD40, CD80, CD86, and MHC-II in monocytes along macrophage differentiation and impair the induction of CD40, CD83, and CD86 during the following maturation $[49,50]$. Second, in mature DCs, some data suggest that MSCs interfere with their phagocytic function and ability to activate T cells [51]. Moreover, cytokines like interleukin-6 (IL-6), PGE2, and macrophage-colony stimulating factor (M-CSF) $[49,51]$ secreted by MSCs can in turn modulate the cytokine profile of DCs. This leads to stimulation of DCs to secrete the anti-inflammatory cytokine IL-10 and to reduce the secretion of interleukin-12 (IL-12) and TNF- $\alpha$, creating an anti-inflammatory environment and furthermore influencing the function of other immune cells [19].

2.5. Neutrophils. The two main effects of MSCs on neutrophils are an increase of their lifespan and inflammatory activity. Consistent results show a reduction of the spontaneous apoptosis rate in both resting and activated neutrophils mediated by MSCs secreting IL-6. Apoptosis in neutrophils is positively linked to production of reactive oxygen species (ROS). Hence, as MSCs have been shown to inhibit ROS production, they lead to decrease inflammatory activity and reduced onset of respiratory burst of neutrophils [52-54].

\section{Clinical Trials}

To date, more than 290 clinical trials involving infusion or transplantation of MSCs have been registered at clinicaltrials.gov, the largest part of which depend on the immunomodulatory properties of MSCs. Most of these trials have not been completed yet, but data collected up to now support the biosafety of MSC transplantation in humans. So far, none of them has reported significant pathological incidences related to transplanted cells. Even if generally they have shown clinical benefits below the expected and immunoregulatory mechanisms are not fully understood, there is a general agreement that therapies based on immunomodulatory features of MSCs aiming to treat a number of immunological disorders that currently have no effective treatments are promising.

Experiments aiming to restore damaged tissues relied first on the ability of MSCs to give rise to mesodermal lineages and eventually also on their immunomodulatory and trophic effects. Despite the success of preclinical studies with MSCs in tissue repair [55] and the fact that most phase I clinical trials have not shown any biosafety issues, further trial phases have obtained modest outcomes. Patients with ischemic stroke have been subjected to a phase I clinical trial, in which MSCs were infused to patients aiming to regenerate infarcted tissue. MSC-treated group showed a slightly better recovery than control group [56] in terms of Modified Rankin Scale values after a 3-year follow-up. Several clinical trials have been initiated since then, including a phase II/III trial in Yonsei University in Seoul (clinicaltrials.gov NCT01392105). Also, MSC-based Prochymal developed by Osiris Therapeutics is currently been tested in a myocardial infarction phase II study (clinicaltrials.gov NCT00877903).
Crohn's disease and ulcerative colitis are inflammatory bowel diseases in which progression impaired immune function plays a key role. Phase I studies have been performed by systemic injection of MSCs in patients that did not respond to conventional treatments; their outcome varies from discrete improvements in Crohn's disease activity index (CDAI) [57] to adverse effects due to a mild allergic reaction to the DMSO used for the cryopreservation of the MSCs [58]. Application of Prochymal in Crohn's disease treatment has also shown to be safe, and a phase III clinical trial has been started (clinicaltrials.gov NCT01233960).

Crohn's disease often results in the formation of perianal fistulae, which can also result from other inflammatory diseases, such as cryptoglandular disease. A phase I trial involved the local transplantation of autologous MSCs from lipoaspirates in five patients to test the feasibility and safety of the system. $75 \%$ of treated fistulae were effectively healed, with no adverse effects reported [59]. A later phase II trial in perianal fistulae both with Crohn's disease origin or cryptoglandular origin was conducted in 49 patients using MSCs from lipoaspirates, with an effectiveness of $71 \%$ and a recurrence of $17.6 \%$ after one-year follow-up regardless of the origin of the fistula [60]. However, in a later multicenter, phase III study focused on complex fistulae not associated with Crohn's disease, the same authors did not find significant differences between the control and MSC-treated groups [61].

Multiple sclerosis (MS) is a neurodegenerative inflammatory disease in which antibodies are produced against myelin sheaths of the brain and spinal cord neurons, leading to a wide variety of neurological disorders. Despite the efficiency of MSC treatment in rodent models of experimental encephalomyelitis (Table 1), an induced disease that mimics MS, clinical trials have not obtained positive results so far. As MS is a complex disease with prolonged onset, some trials have focused their endpoints on specific symptoms as a model of a wider effect. A phase I trial with intrathecally delivered autologous MSCs reported improvement of some visual features [62]. In a more recent phase II clinical trial, MS patients in whom disease involved degeneration of the optic nerve were infused intravenously with autologous MSCs. This trial reports modest improvements in the area of the optic nerve and some visual features, such as visual acuity and minimum angle of resolution [63].

It is widely believed that immune response plays also a crucial role in the development of amyotrophic lateral sclerosis (ALS). ALS is an incurable neurodegenerative disease that progresses rapidly impairing motor neuron function. MSC-based cell therapy has emerged as a promising approach to treat these neurological diseases, due to, on the one hand, their ability to support tissue regeneration and local stem cell stimulation through trophic effects and, on the other hand, their immunomodulatory properties. A third feature of MSCs has been proposed. It is very controversial, which claims that MSCs might have transdifferentiating capacity towards neural lineages [64-66].

A phase I clinical trial was performed in ten ALS patients by injecting MSCs in cerebrospinal fluid. Patients showed no symptoms of adverse effects, although the effectiveness of the treatment could not be assessed [67]. A second phase 
TABLE 1

\begin{tabular}{|c|c|c|c|c|c|}
\hline Pathology & Animal species & Target organ & Mechanism of action & WD & Ref. \\
\hline Lupus erythematosus & Mouse & Bone marrow & Regeneration of hematopoietic niche & Sys & {$[22]$} \\
\hline Rejection of HSC transplantation & Sheep & $\begin{array}{l}\text { Hematopoietic } \\
\text { compartment }\end{array}$ & $\begin{array}{l}\text { Improve transplantation efficiency, increase } \\
\text { hematopoiesis }\end{array}$ & Sys & [23] \\
\hline Rejection of cutaneous grafts & Monkey & Skin & Inhibition of T cells & Sys & [24] \\
\hline Ictus & Rat & $\begin{array}{l}\text { Central nervous } \\
\text { system }\end{array}$ & Secretion of neurotrophic factors & Sys & {$[25]$} \\
\hline Acute renal failure & Rat & Kidneys & $\begin{array}{l}\text { Secretion of trophic factors. Inhibition of } \\
\text { proinflammatory cytokines }\end{array}$ & Sys & [26] \\
\hline Diabetes & Mouse & $\begin{array}{l}\text { Pancreas and renal } \\
\text { glomeruli }\end{array}$ & Inhibition of macrophage infiltration & Sys & [27] \\
\hline Diabetes & Mouse & Pancreas & Inhibition of $\beta$ cell-specific $\mathrm{T}$ cells & Sys & [28] \\
\hline Rejection of transplanted islets & Mouse & Kidney capsule & Inhibition of $\beta$ cell-specific T cells & Loc & [29] \\
\hline Rheumatoid arthritis & Mouse & Joints & $\begin{array}{l}\text { Inhibition of secretion of proinflammatory cytokines } \\
\text { and inhibition of } \mathrm{T} \text { cells }\end{array}$ & Sys & {$[30]$} \\
\hline Retinal degeneration & Rat & Eyes & Induction and secretion of trophic factors & Loc & {$[31]$} \\
\hline Acute lung injury & Mouse & Lungs & Inhibition of secretion of proinflammatory cytokines & Sys & {$[32]$} \\
\hline Acute lung injury & Mouse & Lungs & $\begin{array}{l}\text { Inhibition of secretion of proinflammatory } \\
\text { cytokines. } \\
\text { Secretion of IL-10 }\end{array}$ & Loc & [33] \\
\hline Hepatic failure & Mouse & Liver & Inhibition of inflammatory infiltrate & $\mathrm{CM}$ & {$[34]$} \\
\hline $\begin{array}{l}\text { Experimental autoimmune } \\
\text { encephalomyelitis }\end{array}$ & Rat & $\begin{array}{l}\text { Central nervous } \\
\text { system }\end{array}$ & Inhibition of myelin-specific $\mathrm{T}$ cells & Sys & [35] \\
\hline Myocardial infarction & Rat & Heart & Secretion of trophic factor SFRP2 & Loc & [36] \\
\hline Ulcerative colitis & Mouse & Gut & $\begin{array}{l}\text { Suppression of inflammatory infiltrates and } \\
\text { cytokines. Increase of regulatory T cell activity }\end{array}$ & Sys & [37] \\
\hline
\end{tabular}

I study, involving both ALS $(n=19)$ and MS $(n=15)$ patients, was performed with intrathecally or intravenously delivered magnetically labeled MSCs. Mild adverse effects, including transient fever and headache, were attributed to the injection. Data suggested that a MSCs population had migrated to meninges, subarachnoid space and spinal cord. Regarding immunomodulatory outcome, MSC transplantation resulted in an increase in $\mathrm{CD} 4{ }^{+} \mathrm{CD} 25^{+}$Foxp $3^{+}$regulatory $\mathrm{T}$ cells and a downregulation of activated lymphocytes and antigen-presenting cells [68]. These treatments have shown no biosafety issues, so several phase II trials have been initiated.

Diabetes is also a promising target for MSC-based therapies, due to its local, autoimmune nature and its high prevalence and severity. MSCs' ability to suppress autoimmunity against islets, help damaged islet regeneration, and enhance survival of engrafted islets in mouse diabetes models (Table 1) has encouraged the initiation of a number of phase I-II clinical trials, most of which are still ongoing. In the clinicaltrials.gov database, more than 20 trials involving MSCs, adipose-derived cells, or Prochymal for treatment of diabetes are listed.

Graft-versus-host disease (GVHD) is, at least in theory, one of the most suitable candidates for MSC-based applications. In its acute form, this systemic immune reaction prompted by grafted immune cells can be very severe and is often refractory to classical immunosuppressive treatments. The treatment of GVHD by MSC transplantation has successfully overcome phase I and II clinical trials. A pioneer compassionate study involving only one patient showed a complete response after 1 year [69]. In a later compassionate use study, Prochymal was delivered to twelve pediatric patients suffering severe acute GVHD refractory to immunosuppressive treatments. Severe acute refractory GVHD has been reported to have an overall 2-year survival of 5\%-30\% [70]. Overall, infusions were well tolerated and no acute toxicity was observed. Regarding effectiveness, even if some patients had only partial response to treatment, the overall surviving rate after a median of 611 days rose up to 42\% (five patients) [71]. Another phase II study has been performed with this product in grade II-IV patients, with promising response and survival rates and no adverse effects reported. In this study, conventional steroid and mycophenolate mofetil therapy was supplemented with Prochymal treatment; $77 \%$ of patients achieved a complete response (CR), while steroid therapy alone usually leads to a CR rate of 10-35\%. In patients achieving CR the survival rate at day 90 was $88 \%$ [72]. Currently, GVHD treatment using Prochymal is being tested in two phase III studies by Osiris, one of them involving newly diagnosed patients (NCT00562497) and the other one aiming to treat steroid-refractory GVHD (NCT00366145). Preliminary results announced by press release include a slight improvement in response and durable CR rates specifically in liver GVHD and gastrointestinal GVHD steroid-refractory patients and an overall trend of better response rates in pediatric patients [73]. In 2012, Health 
Canada and Medsafe New Zealand approved Prochymal as their first stem cell therapy for GVDH in children under 18 [74]. Besides, a phase II study using MSCs derived by the European Group for Blood and Marrow Transplantation ex vivo procedure involved 55 severe acute GVHD patients and showed an improvement of 2-year survival rate (53\% versus $26 \%$ of uninfused patients), in the absence of side effects [75].

Although GVHD clinical trials have pioneered the use of MSC-mediated immunosuppression to avoid rejection of allografts, increasing numbers of MSC-based clinical trials are being initiated aiming to improve transplanted solid organ tolerance. Experiments in mice have shown that MSCs can increase immune tolerance for allografts (Table 1), and several clinical trials have been started since then. Mostly, MSCs are regarded as a strategy to reduce immunosuppressant pharmacotherapy doses, as transplantation success is often linked to drug-induced secondary effects. A pilot study has recently suggested that MSC delivery after kidney transplant is safe and allows reduction of tacrolimus dose to a half [76]. Another study has suggested that MSC treatment also allows reduction of the dose of calcineurin inhibitors (CNI), which are administered to reduce acute rejection reactions. In that clinical trial, patients receiving lower CNI doses plus MSC infusion had similar rejection rates after kidney transplant, and slightly better renal function recovery [77]. Furthermore, several phase I-II clinical trials with MSCs are ongoing in liver transplant. However, the outcomes are not available yet.

Despite the very optimistic results obtained in mouse models, MSC-based preliminary clinical trials have not fully met the expectations. The lack of obvious outcomes in clinical trials may be the result of specific human MSCs features and MSC-niche interactions and must be further addressed by analyzing these factors in human contexts. Indeed, there is renewed interest in studying these specific interaction processes in order to boost the therapeutic effect of MSCs. Examples of this effort are genetic manipulation of MSCs or in vitro priming of MSCs cultures, which is discussed below.

\section{Priming MSC-Mediated Immunosuppression}

The ability of MSCs to modulate the activity of surrounding cells is not constitutive but rather requires activation by signals from a proinflammatory environment [20]. This process, termed priming or licensing, is very complex, and little is known about all the factors and signaling pathways involved [9]. In the last years, several papers have been published revealing the role of interleukin-1 (IL-1), IFN- $\gamma$, and TNF- $\alpha$ in this process $[16,20,78]$.

Although the precise signaling pathway involved in the priming by IFN- $\gamma$ is unknown, different authors describe that IFN- $\gamma$ activates the transcription and synthesis of IDO-1 and increases expression levels of HGF and TGF- $\beta$ in MSCs $[16,78]$. In addition, IFN- $\gamma$ stimulation of MSCs renders them resistant to activated NK-mediated attack, thereby allowing them to survive in the inflammatory environment [44]. In mouse models of colitis, the stimulation of human
MSCs in vitro with IFN- $\gamma$ before the transplantation yields better curative results [79]. These experiments reveal that priming by IFN- $\gamma$ can increase the therapeutic effect of transplanted MSCs and points out a concept that should be taken into consideration in the design of clinical trials. However, it has been described that low concentrations of IFN- $\gamma$ promote antigen-presenting cell function on MSCs and exert a proinflammatory effect [80]. This data should be evaluated carefully if this methodology is going to be implemented in future clinical trials.

IL-1 $\beta$ is another cytokine linked to MSC priming [20]. Secreted by macrophages in the inflammatory environment, this cytokine not only activates the secretion of immunosuppressive factors in MSCs but also increases migration of MSCs and the secretion of leukocyte-chemotactic factors. Unlike the stimulation of MSC with IFN- $\gamma$, it is known that IL- $1 \beta$ exerts its effect partially through the activation of NF- $\kappa \mathrm{B}$ signaling [81]. In this sense, it has been published that TNF- $\alpha$, a proinflammatory cytokine secreted by $\mathrm{T}$ cells, NK cells, and macrophages, induces the expression of various proteins related to immunosuppression and increases the migration of stromal cells [82-84].

The fact that these proinflammatory cytokines and signaling pathways can also activate MSC-mediated immunosuppression may seem paradoxical. When the inflammatory reaction overshoots, the same signals that are normally involved in inflammation prime the MSCs and activate their immunomodulatory properties as a negative feedback safety loop. In this regard, the overload of proinflammatory cytokines is precisely the necessary step that activates immunosuppression in MSCs in order to avoid a nondesired immunosuppression due to resident stromal cells. Moreover, in the absence of IFN- $\gamma$ signaling, NK cells are able to kill MSCs, as an additional safety mechanism [85].

\section{In Conclusion}

Cell-based therapy employing MSCs has evolved as an interesting approach in the treatment of a wide range of autoimmune disorders as well as graft rejection, and a large number of clinical trials are currently ongoing. Although there are promising results the outcome has not fully met the expectations from preceding experiments in mouse models. This could be due to the differences observed between mechanisms of human and murine MSC immunoregulations. Furthermore, it is known that the immunomodulatory response changes depend on the given inflammatory environment. Increased knowledge about the complex crosstalk between MSCs and the immune system in humans could help to find clues about how to improve the therapeutic effect of MSCs. In the last years, cell priming has become an emerging research area in the field of MSCs immunomodulation. The encouraging results obtained aiming to enhance the immunoregulatory properties of MSCs render primed and genetically modified MSCs interesting alternatives worth being considered in future clinical trials. Additionally, analysis of data from clinical trials will be needed to optimize treatment dose, timing and frequency of administration. 


\section{Conflict of Interests}

The authors declare that there is no conflict of interests.

\section{References}

[1] A. J. Friedenstein, U. F. Deriglasova, N. N. Kulagina et al., "Precursors for fibroblasts in different populations of hematopoietic cells as detected by the in vitro colony assay method," Experimental Hematology, vol. 2, no. 2, pp. 83-92, 1974.

[2] M. F. Pittenger, "Multilineage potential of adult human mesenchymal stem cells," Science, vol. 284, no. 5411, pp. 143-147, 1999.

[3] M. Dominici, K. Le Blanc, I. Mueller et al., "Minimal criteria for defining multipotent mesenchymal stromal cells. The international society for cellular therapy position statement," Cytotherapy, vol. 8, no. 4, pp. 315-317, 2006.

[4] X. Chen, M. A. Armstrong, and G. Li, "Mesenchymal stem cells in immunoregulation," Immunology and Cell Biology, vol. 84, no. 5, pp. 413-421, 2006.

[5] F. Dazzi and M. Krampera, "Mesenchymal stem cells and autoimmune diseases," Best Practice and Research: Clinical Haematology, vol. 24, no. 1, pp. 49-57, 2011.

[6] M. Krampera, "Mesenchymal stromal cells: more than inhibitory cells," Leukemia, vol. 25, no. 4, pp. 565-566, 2011.

[7] O. Delarosa, E. Lombardo, A. Beraza et al., "Requirement of IFN- $\gamma$-mediated indoleamine 2,3-dioxygenase expression in the modulation of lymphocyte proliferation by human adiposederived stem cells," Tissue Engineering A, vol. 15, no. 10, pp. 2795-2806, 2009.

[8] K. English, F. P. Barry, C. P. Field-Corbett, and B. P. Mahon, "IFN- $\gamma$ and TNF- $\alpha$ differentially regulate immunomodulation by murine mesenchymal stem cells," Immunology Letters, vol. 110, no. 2, pp. 91-100, 2007.

[9] M. Krampera, "Mesenchymal stromal cell licensing: a multistep process," Leukemia, vol. 25, no. 9, pp. 1408-1414, 2011.

[10] G. Ren, L. Zhang, X. Zhao et al., "Mesenchymal stem cellmediated immunosuppression occurs via concerted action of chemokines and nitric oxide," Cell Stem Cell, vol. 2, no. 2, pp. 141-150, 2008.

[11] K. Le Blanc, L. Tammik, B. Sundberg, S. E. Haynesworth, and O. Ringdén, "Mesenchymal stem cells inhibit and stimulate mixed lymphocyte cultures and mitogenic responses independently of the major histocompatibility complex," Scandinavian Journal of Immunology, vol. 57, no. 1, pp. 11-20, 2003.

[12] M. D. Nicola, C. Carlo-Stella, M. Magni et al., "Human bone marrow stromal cells suppress T-lymphocyte proliferation induced by cellular or nonspecific mitogenic stimuli," Blood, vol. 99, no. 10, pp. 3838-3843, 2002.

[13] S. Glennie, I. Soeiro, P. J. Dyson, E. W. Lam, and F. Dazzi, "Bone marrow mesenchymal stem cells induce division arrest anergy of activated T cells," Blood, vol. 105, no. 7, pp. 2821-2827, 2005.

[14] P. Hwu, M. X. Du, R. Lapointe, M. Do, M. W. Taylor, and H. A. Young, "Indoleamine 2,3-dioxygenase production by human dendritic cells results in the inhibition of T cell proliferation," Journal of Immunology, vol. 164, no. 7, pp. 3596-3599, 2000.

[15] D. H. Munn, E. Shafizadeh, J. T. Attwood, I. Bondarev, A. Pashine, and A. L. Mellor, "Inhibition of T cell proliferation by macrophage tryptophan catabolism," Journal of Experimental Medicine, vol. 189, no. 9, pp. 1363-1372, 1999.
[16] J. M. Ryan, F. Barry, J. M. Murphy, and B. P. Mahon, "Interferon$\gamma$ does not break, but promotes the immunosuppressive capacity of adult human mesenchymal stem cells," Clinical and Experimental Immunology, vol. 149, no. 2, pp. 353-363, 2007.

[17] R. Meisel, A. Zibert, M. Laryea, U. Göbel, W. Däubener, and D. Dilloo, "Human bone marrow stromal cells inhibit allogeneic T-cell responses by indoleamine 2,3-dioxygenasemediated tryptophan degradation," Blood, vol. 103, no. 12, pp. 4619-4621, 2004.

[18] D. Sakata, C. Yao, and S. Narumiya, "Prostaglandin E2, an immunoactivator," Journal of Pharmacological Sciences, vol. 112, no. 1, pp. 1-5, 2010.

[19] S. Aggarwal and M. F. Pittenger, "Human mesenchymal stem cells modulate allogeneic immune cell responses," Blood, vol. 105, no. 4, pp. 1815-1822, 2005.

[20] M. E. Groh, B. Maitra, E. Szekely, and O. N. Koç, "Human mesenchymal stem cells require monocyte-mediated activation to suppress alloreactive T cells," Experimental Hematology, vol. 33, no. 8, pp. 928-934, 2005.

[21] K. Le Blanc, I. Rasmusson, C. Götherström et al., "Mesenchymal stem cells inhibit the expression of CD25 (interleukin-2 receptor) and CD38 on phytohaemagglutinin-activated lymphocytes," Scandinavian Journal of Immunology, vol. 60, no. 3, pp. 307-315, 2004.

[22] L. Sun, K. Akiyama, H. Zhang et al., "Mesenchymal stem cell transplantation reverses multiorgan dysfunction in systemic lupus erythematosus mice and humans," Stem Cells, vol. 27, no. 6, pp. 1421-1432, 2009.

[23] G. Almeida-Porada, C. D. Porada, N. Tran, and E. D. Zanjani, "Cotransplantation of human stromal cell progenitors into preimmune fetal sheep results in early appearance of human donor cells in circulation and boosts cell levels in bone marrow at later time points after transplantation," Blood, vol. 95, no. 11, pp. 3620-3627, 2000.

[24] A. Bartholomew, C. Sturgeon, M. Siatskas et al., "Mesenchymal stem cells suppress lymphocyte proliferation in vitro and prolong skin graft survival in vivo," Experimental Hematology, vol. 30, no. 1, pp. 42-48, 2002.

[25] Y. Li, J. Chen, X. G. Chen et al., "Human marrow stromal cell therapy for stroke in rat: neurotrophins and functional recovery," Neurology, vol. 59, no. 4, pp. 514-523, 2002.

[26] F. Tögel, Z. Hu, K. Weiss, J. Isaac, C. Lange, and C. Westenfelder, "Administered mesenchymal stem cells protect against ischemic acute renal failure through differentiation-independent mechanisms," The American Journal of Physiology-Renal Physiology, vol. 289, no. 1, pp. F31-F42, 2005.

[27] R. H. Lee, M. J. Seo, R. L. Reger et al., "Multipotent stromal cells from human marrow home to and promote repair of pancreatic islets and renal glomeruli in diabetic NOD/scid mice," Proceedings of the National Academy of Sciences of the United States of America, vol. 103, no. 46, pp. 17438-17443, 2006.

[28] V. S. Urbán, J. Kiss, J. Kovács et al., "Mesenchymal stem cells cooperate with bone marrow cells in therapy of diabetes," Stem Cells, vol. 26, no. 1, pp. 244-253, 2008.

[29] Y. Ding, D. Xu, G. Feng, A. Bushell, R. J. Muschel, and K. J. Wood, "Mesenchymal stem cells prevent the rejection of fully allogenic islet grafts by the immunosuppressive activity of matrix metalloproteinase-2 and -9," Diabetes, vol. 58, no. 8, pp. 1797-1806, 2009.

[30] A. Augello, R. Tasso, S. M. Negrini, R. Cancedda, and G. Pennesi, "Cell therapy using allogeneic bone marrow mesenchymal 
stem cells prevents tissue damage in collagen-induced arthritis," Arthritis and Rheumatism, vol. 56, no. 4, pp. 1175-1186, 2007.

[31] Y. Inoue, A. Iriyama, S. Ueno et al., "Subretinal transplantation of bone marrow mesenchymal stem cells delays retinal degeneration in the RCS rat model of retinal degeneration," Experimental Eye Research, vol. 85, no. 2, pp. 234-241, 2007.

[32] L. A. Ortiz, M. DuTreil, C. Fattman et al., "Interleukin 1 receptor antagonist mediates the antiinflammatory and antifibrotic effect of mesenchymal stem cells during lung injury," Proceedings of the National Academy of Sciences of the United States of America, vol. 104, no. 26, pp. 11002-11007, 2007.

[33] N. Gupta, X. Su, B. Popov, J. W. Lee, V. Serikov, and M. A. Matthay, "Intrapulmonary delivery of bone marrow-derived mesenchymal stem cells improves survival and attenuates endotoxin-induced acute lung injury in mice," Journal of Immunology, vol. 179, no. 3, pp. 1855-1863, 2007.

[34] B. Parekkadan, D. van Poll, K. Suganuma et al., "Mesenchymal stem cell-derived molecules reverse fulminant hepatic failure," PLoS ONE, vol. 2, no. 9, article e941, 2007.

[35] E. Gerdoni, B. Gallo, S. Casazza et al., "Mesenchymal stem cells effectively modulate pathogenic immune response in experimental autoimmune encephalomyelitis," Annals of Neurology, vol. 61, no. 3, pp. 219-227, 2007.

[36] M. Mirotsou, Z. Zhang, A. Deb et al., "Secreted frizzled related protein 2 (Sfrp2) is the key Akt-mesenchymal stem cell-released paracrine factor mediating myocardial survival and repair," Proceedings of the National Academy of Sciences of the United States of America, vol. 104, no. 5, pp. 1643-1648, 2007.

[37] Q. Zhang, S. Shi, Y. Liu et al., "Mesenchymal stem cells derived from human gingiva are capable of immunomodulatory functions and ameliorate inflammation-related tissue destruction in experimental colitis," Journal of Immunology, vol. 183, no. 12, pp. 7787-7798, 2009.

[38] A. Gonzalez, V. Rebmann, J. LeMaoult, P. A. Horn, E. D. Carosella, and E. Alegre, "The immunosuppressive molecule HLA-G and its clinical implications," Critical Reviews in Clinical Laboratory Sciences, vol. 49, no. 3, pp. 63-84, 2012.

[39] A. Nasef, N. Mathieu, A. Chapel et al., "Immunosuppressive effects of mesenchymal stem cells: involvement of HLA-G," Transplantation, vol. 84, no. 2, pp. 231-237, 2007.

[40] Z. Selmani, A. Naji, E. Gaiffe et al., "HLA-G is a crucial immunosuppressive molecule secreted by adult human mesenchymal stem cells," Transplantation, vol. 87, no. 9, pp. S62-S66, 2009.

[41] Z. Selmani, A. Naji, I. Zidi et al., "Human leukocyte antigenG5 secretion by human mesenchymal stem cells is required to suppress T lymphocyte and natural killer function and to induce CD4+ CD25highFOXP3+ regulatory T cells," Stem Cells, vol. 26, no. 1, pp. 212-222, 2008.

[42] P. A. Sotiropoulou, S. A. Perez, A. D. Gritzapis, C. N. Baxevanis, and M. Papamichail, "Interactions between human mesenchymal stem cells and natural killer cells," Stem Cells, vol. 24, no. 1, pp. 74-85, 2006.

[43] I. Rasmusson, O. Ringdén, B. Sundberg, and K. Le Blanc, "Mesenchymal stem cells inhibit the formation of cytotoxic T lymphocytes, but not activated cytotoxic T lymphocytes or natural killer cells," Transplantation, vol. 76, no. 8, pp. 1208-1213, 2003.

[44] G. M. Spaggiari, A. Capobianco, S. Becchetti, M. C. Mingari, and L. Moretta, "Mesenchymal stem cell-natural killer cell interactions: evidence that activated NK cells are capable of killing MSCs, whereas MSCs can inhibit IL-2-induced NK-cell proliferation," Blood, vol. 107, no. 4, pp. 1484-1490, 2006.
[45] A. Corcione, F. Benvenuto, E. Ferretti et al., "Human mesenchymal stem cells modulate B-cell functions," Blood, vol. 107, no. 1, pp. 367-372, 2006.

[46] I. Rasmusson, K. Le Blanc, B. Sundberg, and O. Ringdén, "Mesenchymal stem cells stimulate antibody secretion in human B cells," Scandinavian Journal of Immunology, vol. 65, no. 4, pp. 336-343, 2007.

[47] S. Tabera, J. A. Pérez-Simón, M. Díez-Campelo et al., “The effect of mesenchymal stem cells on the viability, proliferation and differentiation of B-lymphocytes," Haematologica, vol. 93, no. 9, pp. 1301-1309, 2008.

[48] E. Traggiai, S. Volpi, F. Schena et al., "Bone marrow-derived mesenchymal stem cells induce both polyclonal expansion and differentiation of $\mathrm{B}$ cells isolated from healthy donors and systemic lupus erythematosus patients," Stem Cells, vol. 26, no. 2, pp. 562-569, 2008.

[49] G. M. Spaggiari, H. Abdelrazik, F. Becchetti, and L. Moretta, "MSCs inhibit monocyte-derived DC maturation and function by selectively interfering with the generation of immature DCs: central role of MSC-derived prostaglandin E2," Blood, vol. 113, no. 26, pp. 6576-6583, 2009.

[50] W. Zhang, W. Ge, C. Li et al., "Effects of mesenchymal stem cells on differentiation, maturation, and function of human monocyte-derived dendritic cells," Stem Cells and Development, vol. 13, no. 3, pp. 263-271, 2004.

[51] A. J. Nauta, A. B. Kruisselbrink, E. Lurvink, R. Willemze, and W. E. Fibbe, "Mesenchymal stem cells inhibit generation and function of both CD34+-derived and monocyte-derived dendritic cells," Journal of Immunology, vol. 177, no. 4, pp. 20802087, 2006.

[52] S. Brandau, M. Jakob, H. Hemeda et al., "Tissue-resident mesenchymal stem cells attract peripheral blood neutrophils and enhance their inflammatory activity in response to microbial challenge," Journal of Leukocyte Biology, vol. 88, no. 5, pp. 10051015, 2010.

[53] M. A. Cassatella, F. Mosna, A. Micheletti et al., "Toll-like receptor-3-activated human mesenchymal stromal cells significantly prolong the survival and function of neutrophils," Stem Cells, vol. 29, no. 6, pp. 1001-1011, 2011.

[54] L. Raffaghello, G. Bianchi, M. Bertolotto et al., "Human mesenchymal stem cells inhibit neutrophil apoptosis: a model for neutrophil preservation in the bone marrow niche," Stem Cells, vol. 26, no. 1, pp. 151-162, 2008.

[55] D. G. Phinney and D. J. Prockop, "Concise review: mesenchymal stem/multipotent stromal cells: the state of transdifferentiation and modes of tissue repair-current views," Stem Cells, vol. 25, no. 11, pp. 2896-2902, 2007.

[56] J. S. Lee, J. M. Hong, G. J. Moon, P. H. Lee, Y. H. Ahn, and O. Y. Bang, "A long-term follow-up study of intravenous autologous mesenchymal stem cell transplantation in patients with ischemic stroke," Stem Cells, vol. 28, no. 6, pp. 1099-1106, 2010.

[57] J. Onken, D. Gallup, and J. Hanson, "Successful outpatient treatment of refractory Crohn's disease using adult mesenchymal stem cells," in Proceedings of the American College of Gastroenterology Annual Meeting, pp. 23-25, 2006.

[58] M. Duijvestein, A. C. W. Vos, H. Roelofs et al., "Autologous bone marrow-derived mesenchymal stromal cell treatment for refractory luminal Crohn's disease: results of a phase I study," Gut, vol. 59, no. 12, pp. 1662-1669, 2010.

[59] D. García-Olmo, M. García-Arranz, D. Herreros, I. Pascual, C. Peiro, and J. A. Rodríguez-Montes, "A phase I clinical trial of 
the treatment of crohn's fistula by adipose mesenchymal stem cell transplantation," Diseases of the Colon and Rectum, vol. 48, no. 7, pp. 1416-1423, 2005.

[60] D. Garcia-Olmo, D. Herreros, I. Pascual et al., "Expanded adipose-derived stem cells for the treatment of complex perianal fistula: a phase II clinical trial," Diseases of the Colon and Rectum, vol. 52, no. 1, pp. 79-86, 2009.

[61] M. D. Herreros, M. Garcia-Arranz, H. Guadalajara, P. DeLa-Quintana, and D. Garcia-Olmo, "Autologous expanded adipose-derived stem cells for the treatment of complex cryptoglandular perianal fistulas: a phase III randomized clinical trial (FATT 1: fistula advanced therapy trial 1) and long-term evaluation," Diseases of the Colon and Rectum, vol. 55, no. 7, pp. 762-772, 2012.

[62] B. Yamout, R. Hourani, H. Salti et al., "Bone marrow mesenchymal stem cell transplantation in patients with multiple sclerosis: a pilot study," Journal of Neuroimmunology, vol. 227, no. 1-2, pp. 185-189, 2010.

[63] P. Connick, M. Kolappan, C. Crawley et al., "Autologous mesenchymal stem cells for the treatment of secondary progressive multiple sclerosis: an open-label phase 2a proof-of-concept study," The Lancet Neurology, vol. 11, no. 2, pp. 150-156, 2012.

[64] D. Woodbury, E. J. Schwarz, D. J. Prockop, and I. B. Black, "Adult rat and human bone marrow stromal cells differentiate into neurons," Journal of Neuroscience Research, vol. 61, no. 4, pp. 364-370, 2000.

[65] C. Krabbe, J. Zimmer, and M. Meyer, "Neural transdifferentiation of mesenchymal stem cells-a critical review," APMIS, vol. 113, no. 11-12, pp. 831-844, 2005.

[66] I. Kassis, A. Vaknin-Dembinsky, and D. Karussis, "Bone marrow mesenchymal stem cells: agents of immunomodulation and neuroprotection," Current Stem Cell Research and Therapy, vol. 6, no. 1, pp. 63-68, 2011.

[67] L. Mazzini, I. Ferrero, V. Luparello et al., "Mesenchymal stem cell transplantation in amyotrophic lateral sclerosis: a phase I clinical trial," Experimental Neurology, vol. 223, no. 1, pp. 229237, 2010.

[68] D. Karussis, C. Karageorgiou, A. Vaknin-Dembinsky et al., "Safety and immunological effects of mesenchymal stem cell transplantation in patients with multiple sclerosis and amyotrophic lateral sclerosis," Archives of Neurology, vol. 67, no. 10, pp. 1187-1194, 2010.

[69] K. Le Blanc, I. Rasmusson, B. Sundberg et al., "Treatment of severe acute graft-versus-host disease with third party haploidentical mesenchymal stem cells," The Lancet, vol. 363, no. 9419, pp. 1439-1441, 2004.

[70] J. Y. Cahn, J. P. Klein, S. J. Lee et al., "Prospective evaluation of 2 acute graft-versus-host (GVHD) grading systems: a joint Société Française de Greffe de Moëlle et Thërapie Cellulaire (SFGM-TC), Dana Farber Cancer Institute (DFCI), and international bone marrow transplant registry (IBMTR) prospective study," Blood, vol. 106, no. 4, pp. 1495-1500, 2005.

[71] V. K. Prasad, K. G. Lucas, G. I. Kleiner et al., "Efficacy and safety of ex vivo cultured adult human mesenchymal stem cells (prochymal) in pediatric patients with severe refractory acute graft-versus-host disease in a compassionate use study," Biology of Blood and Marrow Transplantation, vol. 17, no. 4, pp. 534-541, 2011.

[72] P. Kebriaei, L. Isola, E. Bahceci et al., "Adult human mesenchymal stem cells added to corticosteroid therapy for the treatment of acute graft-versus-host disease," Biology of Blood and Marrow Transplantation, vol. 15, no. 7, pp. 804-811, 2009.
[73] C. R. Mills, "Osiris therapeutics announces preliminary results for prochymal phase III GVHD trials," 2009.

[74] D. Cyranoski, "Canada approves stem cell product," Nature Biotechnology, vol. 30, pp. 571-571, 2012.

[75] K. Le Blanc, F. Frassoni, L. Ball et al., "Mesenchymal stem cells for treatment of steroid-resistant, severe, acute graft-versushost disease: a phase II study," The Lancet, vol. 371, no. 9624, pp. 1579-1586, 2008.

[76] Y. Peng, M. Ke, L. Xu et al., "Donor-derived mesenchymal stem cells combined with low-dose tacrolimus prevent acute rejection after renal transplantation: a clinical pilot study," Transplantation, vol. 95, no. 1, pp. 161-168, 2013.

[77] J. Tan, W. Wu, X. Xu et al., "Induction therapy with autologous mesenchymal stem cells in living-related kidney transplants: a randomized controlled trial," The Journal of the American Medical Association, vol. 307, no. 11, pp. 1169-1177, 2012.

[78] M. Krampera, L. Cosmi, R. Angeli et al., "Role for interferon$\gamma$ in the immunomodulatory activity of human bone marrow mesenchymal stem cells," Stem Cells, vol. 24, no. 2, pp. 386-398, 2006.

[79] M. Duijvestein, M. E. Wildenberg, M. M. Welling et al., "Pretreatment with interferon- $\gamma$ enhances the therapeutic activity of mesenchymal stromal cells in animal models of colitis," Stem Cells, vol. 29, no. 10, pp. 1549-1558, 2011.

[80] J. L. Chan, K. C. Tang, A. P. Patel et al., "Antigen-presenting property of mesenchymal stem cells occurs during a narrow window at low levels of interferon- $\gamma$," Blood, vol. 107, no. 12, pp. 4817-4824, 2006.

[81] R. Carrero, I. Cerrada, E. Lledó et al., "IL1 $\beta$ induces mesenchymal stem cells migration and leucocyte chemotaxis through NF$\kappa$ B," Stem Cell Reviews and Reports, vol. 8, no. 3, pp. 905-916, 2012.

[82] A. Zhang, Y. Wang, Z. Ye, H. Xie, L. Zhou, and S. Zheng, "Mechanism of TNF- $\alpha$-induced migration and hepatocyte growth factor production in human mesenchymal stem cells," Journal of Cellular Biochemistry, vol. 111, no. 2, pp. 469-475, 2010.

[83] J. A. Miettinen, M. Pietilä, R. J. Salonen et al., “Tumor necrosis factor alpha promotes the expression of immunosuppressive proteins and enhances the cell growth in a human bone marrow-derived stem cell culture," Experimental Cell Research, vol. 317, no. 6, pp. 791-801, 2011.

[84] W. Böcker, D. Docheva, W. C. Prall et al., "IKK-2 is required for TNF- $\alpha$-induced invasion and proliferation of human mesenchymal stem cells," Journal of Molecular Medicine, vol. 86, no. 10, pp. 1183-1192, 2008.

[85] A. Uccelli, L. Moretta, and V. Pistoia, "Mesenchymal stem cells in health and disease," Nature Reviews Immunology, vol. 8, no. 9, pp. 726-736, 2008. 


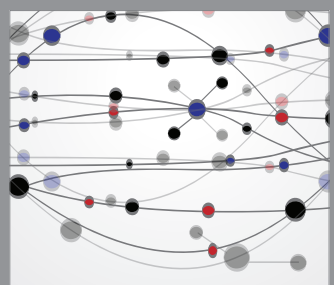

The Scientific World Journal
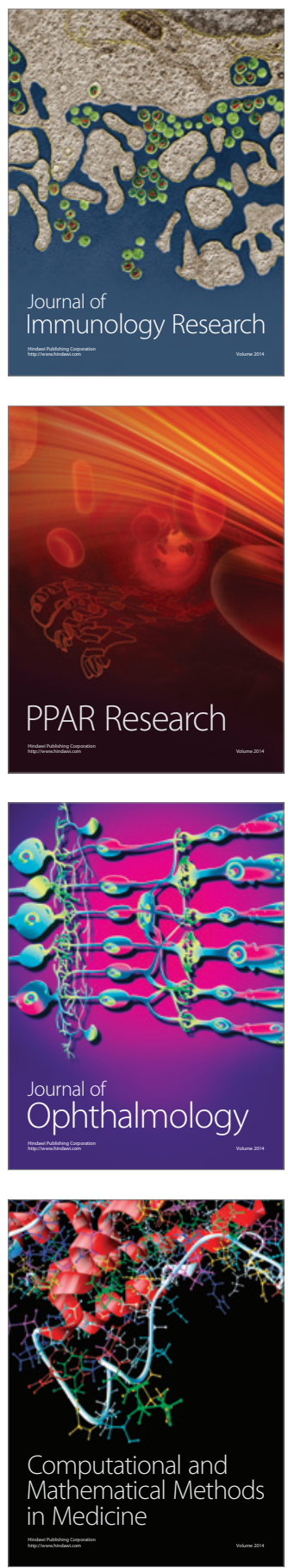

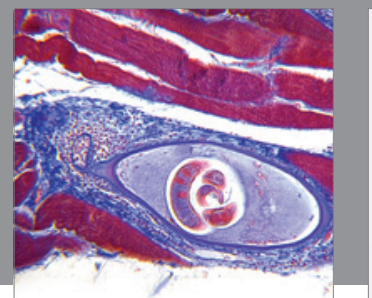

Gastroenterology

Research and Practice
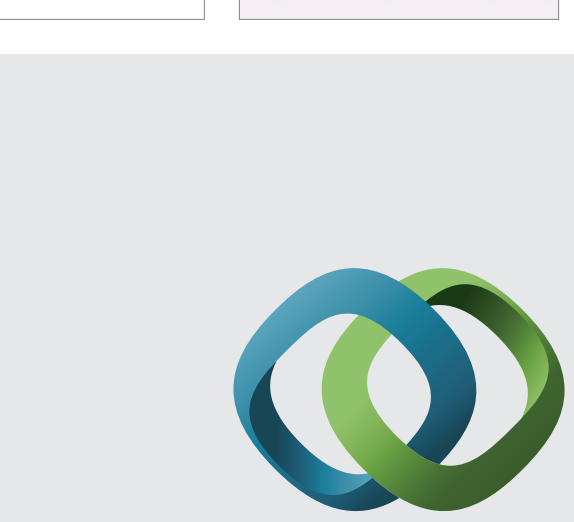

\section{Hindawi}

Submit your manuscripts at

http://www.hindawi.com
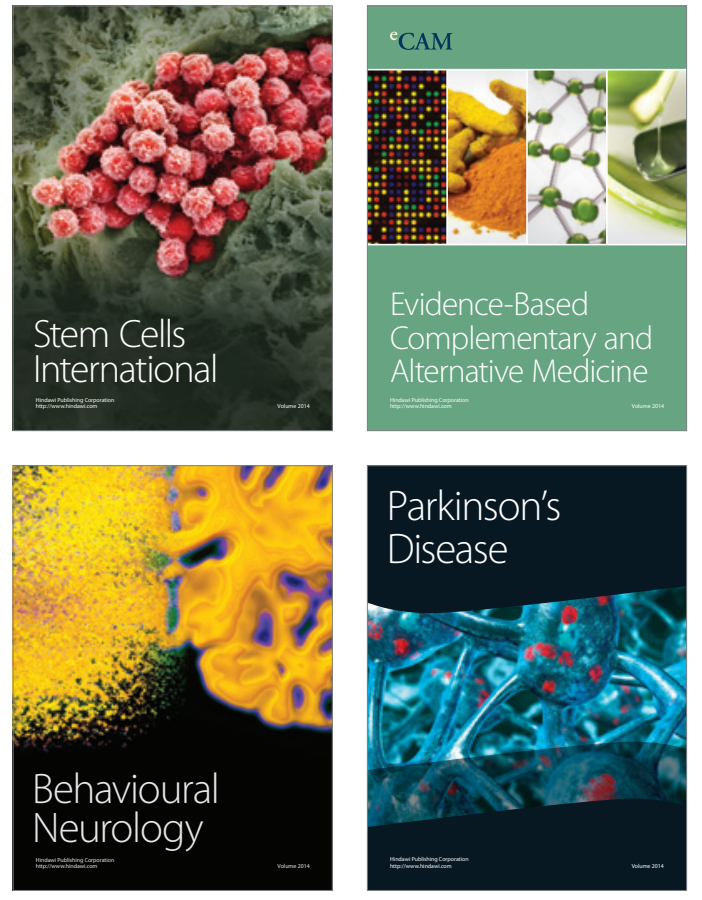
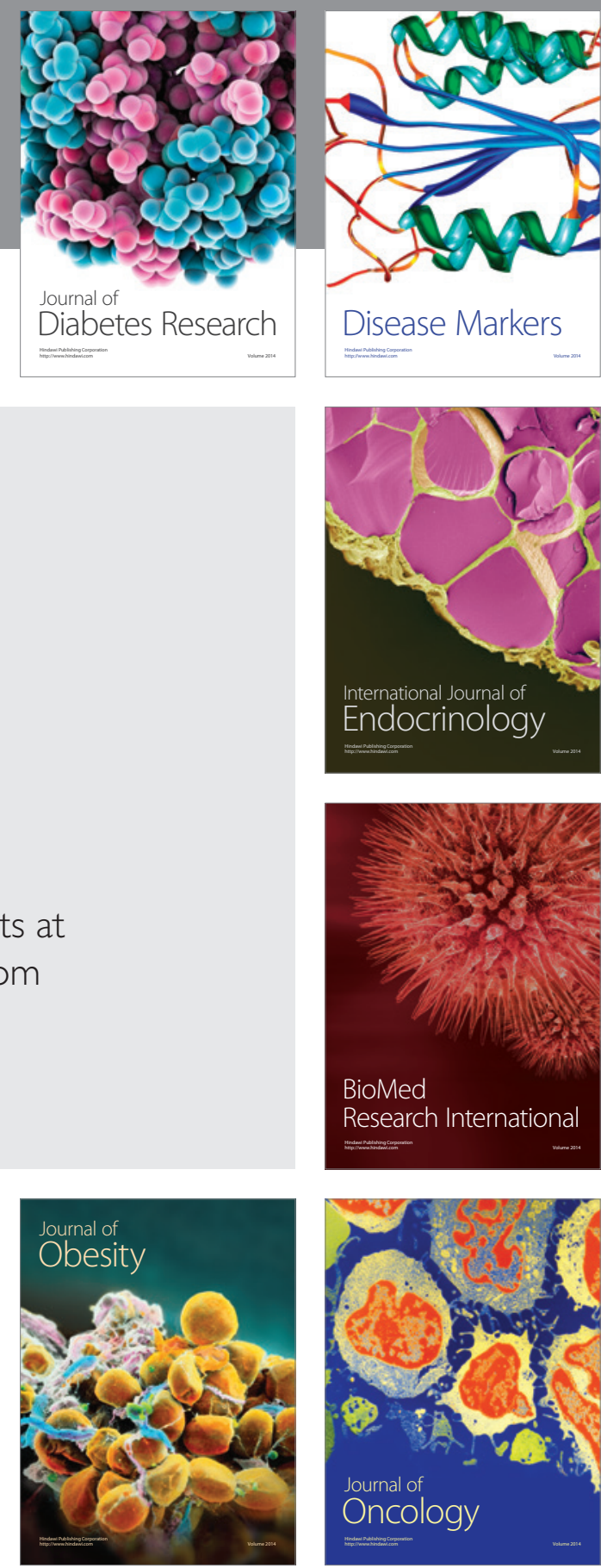

Disease Markers
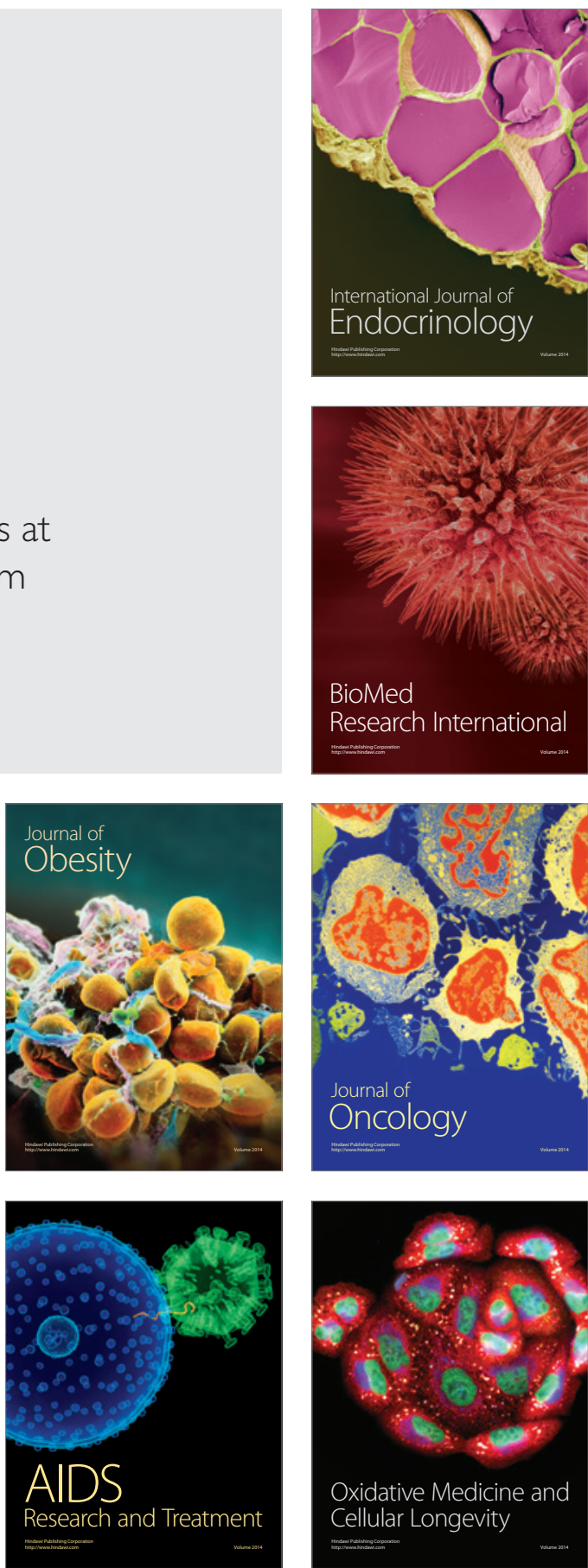